\title{
Ignition Time Delay of Hydrogen-Oxygen-Diluent Mixtures at High Temperatures
}

\author{
J. A. Nrcholls, ${ }^{*}$ T. C. Adamson JR. $\dagger$ And R. B. Morrison $\ddagger$ \\ The University of Michigan, Ann Arbor, Mich.
}

\begin{abstract}
The motivation for this study arose from experiments on standing detonation waves wherein the flame front was visibly separated from the shock front. This physical separation corresponds to the ignition time delay. A theoretical analysis of the ignition time delay zone for hydrogen-oxygen-diluent mixtures is presented herein. A reaction scheme consisting of nine reactions, which should be valid for temperatures above about $1100^{\circ} \mathrm{K}$, is considered. Assuming the initial mole fractions of $\mathrm{H}_{2}$ and $\mathrm{O}_{2}$ to be of order unity and taking advantage of inequalities that should be valid to at least $2000^{\circ} \mathrm{K}$, it is possible to integrate the differential equations representing the kinetics. Thus time dependent expressions for all radical and water vapor concentrations hehind the shock wave are obtained. A characteristic time, based on the initial concentration of $\mathrm{H}_{2}$ and the fastest reaction rate constant, is introduced. For times much greater than this characteristic time, the equations reduce to very simple forms. In order to arrive at an explicit analytical expression for the ignition time delay, a value for the mole fraction of $\mathrm{H}$, characteristic of this delay time, is introduced. It is found that the delay time is dependent on the temperature, pressure, composition, and reaction rate constant for the rate controlling reaction. There is weak dependence on the reaction rate constants for the initiation reactions. A brief comparison between this theory and some experimental results on standing detonation waves is presented and good agreement found. The theory is also in good agreement with available shock tube results.
\end{abstract}

\section{Introduction}

$\mathbf{T}$ $\mathrm{HE}$ results of experiments on standing detonation waves with hydrogen-air mixtures at the University of Michigan have consistently revealed a distinct separation between the shock wave and flame front. This separation corresponds to the ignition time delay. In view of these results, it appeared essential and opportune to consider this facet theoretically and to attempt to corroborate the experimental observations. Such knowledge is of great value to the understanding of the structure of a detonation wave. In addition, it is well to assess the potential value of this steady flow experimental technique to the study of chemical kinetics. Accordingly, a theoretical and experimental investigation of the ignition time delay of hydrogen-oxygen mixtures under detonation or near detonation conditions was effected. The results of these investigations have been published in part. ${ }^{1,2,10,11}$ The theoretical approach presented herein represents a more rigorous treatment of the pertinent reaction equations.

The problem to be considered is that wherein a hydrogenoxygen-diluent mixture is subjected to a normal shock wave sufficiently strong to cause ignition. It is known (Semenov ${ }^{3}$ and Lewis and von Elbe ${ }^{4}$ ) that under these conditions an

Received by IAS October 29, 1962; revision received July 22 , 1963. This research was supported by the U. S. Air Force under Contract AF 49(638)-562, monitored by the Air Force Office of Scientific Research, Propulsion Research Division, Office of Aerospace Research. The material presented here also appears in Ref. 11. The authors are indebted to Eliahou K. Dabora, a fellow researcher on the study of standing detonation waves, for many enlightening discussions and his strong participation in the experimental portion alluded to in the comparison between theoretical and experimental results.

* Associate Professor and Supervisor of the Aircraft Propulsion Laboratory, Department of Aeronautical and Astronautical Engineering. Member AIAA.

$\dagger$ Professor, Department of Aeronautical and Astronautical Engineering. Member AIAA.

$\ddagger$ Professor, Department of Aeronautical and Astronautical Engineering, currently on leave of absence with NASA. Member AIAA. induction period must exist before any explosion can materialize. Duff ${ }^{5}$ clarified the problem by calculating numerically the reaction profile of a $2 \mathrm{H}_{2}+\mathrm{O}_{2}+X_{e}$ mixture behind a steady state shock wave for a given Mach number, temperature, and pressure. His results for the particular conditions considered indicated that the well-known hydrogen-oxygen chain branching mechanism was established almost immediately. Thus, the induction period in the case of high temperatures represents the time required by the action of the chain to establish radical concentrations and hence rates of reaction which are great enough to affect the thermodynamic and hydrodynamic variables. Once this condition is attained, the temperature rises rapidly and the flame is established. Schott and Kinsey ${ }^{6}$ performed calculations similar to those of Duff and also measured the OH concentration in the shock wave induced reaction of argon diluted hydrogen-oxygen mixtures. They interpreted their ignition delay data in terms of the rate controlling reaction for the branched chain mechanism, the primary initiation reaction, and the time required for the $\mathrm{OH}$ concentration to attain a certain value. In this paper Duff's comprehensive reaction scheme is treated analytically with major emphasis placed on the time dependent description of the hydrogen atom concentration. A physically plausible condition is then invoked which allows prediction of the ignition delay time. The results of this analysis will be compared with some experimentally measured delays.

The problem will be generalized to the extent of including reactions of the type, $x \mathrm{H}_{2}+y \mathrm{O}_{2}+z B \rightarrow$ products, where $B$ is some unspecified inert gas. Hence, various hydrogenoxygen concentrations as well as diluent concentrations will be considered. It will be assumed that no chemical reaction occurs across the shock wave and further that the gases are equilibrated immediately in the rotational and translational degrees of freedom. Realizing that shock waves are only a few mean free paths in thickness, that only $3-5$ and $15-300$ molecular collisions are required to equilibrate the translational and rotational degrees, respectively, and that many thousands of collisions are required generally to complete a chemical reaction, it would appear that the assumptions are perfectly justifiable. Supporting arguments for these 
conclusions are given by Bethe and Teller, ${ }^{7}$ Wood, ${ }^{8}$ and many others. The question of relative times for ignition delay and vibrational relaxation is not well established, as has been discussed by Schott and Kinsey ${ }^{6}$ and Patch. ${ }^{9}$ Evidently there is the distinct possibility that for certain hydrogenoxygen-nitrogen mixtures the vibrational relaxation time may be much shorter than, much greater than, or of the same order as the ignition time delay. In the following theoretical development, it will be assumed that the vibrational relaxation time is either much shorter or much longer than the ignition time delay, so that a temperature may be defined readily without ambiguity. It will not be necessary to assert which of the two cases prevails until one attempts to determine the temperature and pressure behind the shock in terms of the upstream conditions. This problem will arise in a later part of this paper when the experimental results are compared with theory. At that point it will be assumed that vibration is not excited behind the wave, that is, that the vibrational relaxation time is much longer than the ignition delay time.

Throughout the induction zone it is safe to assume no change in the total number of moles per cubic centimeter (i.e., $[C]=$ const), inasmuch as the total number of moles of radicals and $\mathrm{H}_{2} \mathrm{O}$ formed is relatively small. Similarly, the mole fractions of $\mathrm{H}_{2}$ and $\mathrm{O}_{2}$ will remain almost constant, although this condition will be relaxed subsequently in the late stages of the induction period. For the same reason (changes only on the microscopic level), the thermodynamic coordinates and hydrodynamic variables remain fixed so that it is possible to divorce the chemical kinetics from the fluid mechanics. This, of course, greatly simplifies the problem.

\section{Pertinent Reactions and Rates of Reaction}

Some of the more important reactions characterizing the combustion of $\mathrm{H}_{2}-\mathrm{O}_{2}$ mixtures have been postulated by various authors (cf., Semenov ${ }^{3}$ and Lewis and von Elbe ${ }^{4}$ ). There has not been complete agreement on all of these reactions. Duff $^{5}$ considered nine reactions and calculated, by iterative techniques, the reaction profile behind a shock wave moving through a $2 \mathrm{H}_{2}+\mathrm{O}_{2}+X_{e}$ mixture such that the pressure and temperature immediately downstream of the shock were $1.150 \mathrm{~atm}$ and $1737^{\circ} \mathrm{K}$, respectively. For this purpose he used the best known values of the rate constants. Inasmuch as Duff's reaction scheme appears to be the most comprehensive and is in the temperature range of interest here $\left(1100-2000^{\circ} \mathrm{K}\right)$, his proposed reactions will be considered in this work. The aim will be to reduce the reaction scheme to a form that can be handled analytically so that functional information can be gained.

The reactions to be considered are given in Table 1 . The reactions $\mathrm{H}+\mathrm{O}_{2}+M \rightarrow \mathrm{HO}_{2}+M$ along with two other consequent reactions given by Patch $^{9}$ are not included in that they would be of importance only for temperatures below about $1100^{\circ} \mathrm{K}$. Consequently, the subsequent predictions

Table 1 Pertinent reactions in the ignition delay zone

\begin{tabular}{rc}
\hline \hline No. & Reaction \\
\hline $\mathrm{I}$ & $\mathrm{H}_{2} \mathrm{O}+M^{a} \stackrel{k_{1}}{\rightarrow} \mathrm{OH}+M+\mathrm{H}$ \\
$\mathrm{II}$ & $\mathrm{H}_{2}+M \stackrel{k_{2}}{\rightarrow} 2 \mathrm{H}+M$ \\
III & $\mathrm{O}_{2}+M \stackrel{k_{3}}{\rightarrow} 20+M$ \\
IV & $\mathrm{OH}+M \stackrel{k_{4}}{\rightarrow} \mathrm{O}+\mathrm{H}+M$ \\
V & $\mathrm{O}_{2}+\mathrm{H}_{2} \stackrel{k_{5}}{\rightarrow} 2 \mathrm{OH}$ \\
VI & $\mathrm{O}_{2}+\mathrm{H} \stackrel{k_{6}}{\rightarrow} \mathrm{OH}+\mathrm{O}$ \\
VII & $\mathrm{H}_{2}+\mathrm{O} \stackrel{k_{7}}{\rightarrow} \mathrm{OH}+\mathrm{H}$ \\
VIII & $\mathrm{H}_{2}+\mathrm{OH} \underset{k_{8}}{\rightarrow} \mathrm{H}_{2} \mathrm{O}+\mathrm{H}$ \\
IX & $2 \mathrm{OH} \stackrel{k_{9}}{\rightarrow} \mathrm{H}_{2} \mathrm{O}+\mathrm{O}$ \\
\hline
\end{tabular}

a $M=$ some third body. of ignition time delay can be expected to hold only for higher temperatures.

The reactions have been written as proceeding only in the forward direction. This is legitimate for the induction period in that the gas immediately behind the shock is far removed from chemical equilibrium, and hence the reverse reaction rates will not have to be taken into account.

The reaction rate constant $k_{i}$ (the subscript $i$ referring to the particular reaction in Table 1 ) is given by

$$
k_{i}=A_{i} T^{m_{i}} \exp \left(-E_{i} / R T\right)
$$

The values of $A_{i}, m_{i}$, and $E_{i}$ are given by Duff. ${ }^{5}$

In writing the individual rate equations, it is convenient to employ the mole fractions for the species concentrations, i.e., $n_{\mathrm{H}}=[\mathrm{H}] /[C], n_{\mathrm{O}}=[\mathrm{O}] /[C]$, ete. In comparing the importance of individual terms of the rate equations, it is noted that $n_{\mathrm{O}_{2}}$ and $n_{\mathrm{H}_{2}}$ are of order unity, written as $\mathrm{O}\left(n_{\mathrm{O} 2}\right)=$ 1 and $\mathrm{O}\left(n_{\mathrm{H}_{2}}\right)=1$. The concentrations of all radicals are much less than that of $\mathrm{H}_{2}$ and $\mathrm{O}_{2}$ so that

$$
n_{\mathrm{H}} \ll 1 \quad n_{\mathrm{H}_{2} \mathrm{O}} \ll 1 \quad n_{\mathrm{OH}} \ll 1 \quad n_{\mathrm{O}} \ll 1
$$

In view of these inequalities and numerical values of the rate constants, it is possible to eliminate some terms from the rate equations immediately.

Defining the constants as

$$
\begin{array}{ll}
a=[C] k_{1} & f=n_{\mathrm{O}_{2}}[C] k_{6} \\
b=2 n_{\mathrm{H}_{2}}[C] k_{2} & g=n_{\mathrm{H}_{2}}[C] k_{7} \\
c=2 n_{\mathrm{O}_{2}}[C] k_{3} & h=n_{\mathrm{H}_{2}}[C] k_{8} \\
\epsilon=2 n_{\mathrm{O}_{2}} n_{\mathrm{H}_{2}}[C] k_{5} & i=[C] k_{9}
\end{array}
$$

the resultant rate equations may be written

$$
\begin{gathered}
d n_{\mathrm{H}} / d t=\dot{n}_{\mathrm{H}}=b-f n_{\mathrm{H}}+g n_{\mathrm{O}}+h n_{\mathrm{OH}} \\
\dot{n}_{\mathrm{O}}=c+f n_{\mathrm{H}}-g n_{\mathrm{O}}+i n_{\mathrm{OH}^{2}} \\
\dot{n}_{\mathrm{OH}}=\epsilon+f n_{\mathrm{H}}+g n_{\mathrm{O}}-h n_{\mathrm{OH}} \\
\dot{n}_{\mathrm{H}_{2} \mathrm{O}}=-a n_{\mathrm{H}_{2} \mathrm{O}}+h n_{\mathrm{OH}} \\
\dot{n}_{\mathrm{O}_{2}}=-\frac{1}{2} \epsilon-f n_{\mathrm{H}} \\
\dot{n}_{\mathrm{H} 2}=-\frac{1}{2} \epsilon-g n_{\mathrm{O}}-h n_{\mathrm{OH}}
\end{gathered}
$$

These equations are valid throughout the entire induction zone, but at different times different terms predominate.

\section{Solution of the Equations}

The nonlinear term of Eq. (2) may be dropped because at the initial stages of the ignition delay zone it is small compared to $c$, and at the later stages it is small compared to $f n_{\mathrm{H}}$ and $g n_{0}$. This step will be discussed in a subsequent section.

The first three equations then become linear in the three unknowns $n_{\mathrm{H}}(t), n_{\mathrm{O}}(t)$, and $n_{\mathrm{OH}}(t)$ and hence can be solved readily. Introducing the linear transformations

$$
\xi=n_{\mathrm{K}}-\delta_{1} \quad \eta=n_{\mathrm{O}}-\delta_{2} \quad \zeta=n_{\mathrm{OH}}-\delta_{3}
$$

and choosing the constants correctly, the equations become

$$
\begin{gathered}
\dot{\xi}=-f \xi+g \eta+h \zeta \\
\dot{\eta}=f \xi-g \eta \\
\dot{\zeta}=f \xi+g \eta-h \zeta
\end{gathered}
$$

The solutions are

$$
\begin{aligned}
& \xi=\sum_{i=1}^{3} a_{i} e^{\lambda_{i} t} \\
& \eta=\sum_{i=1}^{3} a_{i} b_{i} e^{\lambda_{i} t} \\
& \zeta=\sum_{i=1}^{3} a_{i} c_{i} e^{\lambda_{i}}
\end{aligned}
$$


The quantities $\lambda_{i}, b_{i}$, and $c_{i}$ may be expressed in terms of the $a_{i}$ 's by substitution of relations (10) into (7-9). Then the $a_{i}$ 's are evaluated from the boundary conditions, i.e., at

$$
t=0 \quad n_{\mathrm{H}}=n_{\mathrm{O}}=n_{\mathrm{HO}}=0
$$

In arriving at the final forms, the inequalities $f \ll h$ and $\dot{g} \simeq$ $h$ (but $g \neq h$ ) were used. These inequalities are good for the values of reaction rate constants used, as long as the order of magnitude of $n_{\mathrm{O}_{2}}$ and $n_{\mathrm{H}_{2}}$ is the same.

The resultant expressions for the concentrations of radicals for all $t$, neglecting the very small terms, are

$$
\begin{gathered}
n_{\mathrm{H}}(t)=\frac{b+\epsilon}{2 f}\left(e^{2 f t}-1\right)+\frac{\epsilon}{h} e^{-h t} \\
n_{\mathrm{O}}(t)=\frac{b+\epsilon}{2 g}\left(e^{2 f t}-1\right)-\frac{\epsilon f}{h(h-g)} e^{-h t}+\frac{\epsilon f}{g(h-g)} e^{-g t} \\
n_{\mathrm{OH}}(t)=-\frac{b+c}{h}+\frac{b+\epsilon}{h} e^{2 f t}-\frac{\epsilon}{h} e^{-h t}+\frac{\epsilon f}{(h-g)^{2}} e^{-g t} \\
n_{\mathrm{H}_{2} \mathrm{O}}(t)=\frac{b+\epsilon}{2 f}\left(e^{2 f t}-1\right)+\frac{\epsilon}{h} e^{-h t}=n_{\mathrm{H}}
\end{gathered}
$$

It is convenient at this point to introduce a characteristic time, $t_{c}=1 / h=1 / n_{\mathrm{H}_{2}}[C] k_{8}$, which is based on the initial hydrogen mole fraction, the total concentration, and the fastest reaction rate constant. Also define a dimensionless time:

$$
t^{\prime}=t / t_{c}=h t
$$

The characteristic time is much less than the ignition time delay so it is in order to rewrite Eqs. (11-14) for $t^{\prime} \gg 1$. They are

$$
\begin{gathered}
n_{\mathrm{H}}(t)=[(b+\epsilon) / 2 f]\left(e^{2 f t}-1\right) \\
n_{\mathrm{O}}(t)=[(b+\epsilon) / 2 g]\left(e^{2 f t}-1\right)=(f / g) n_{\mathrm{H}} \\
n_{\mathrm{OH}}(t)=[(b+\epsilon) / h] e^{2 f t}=(2 f / h) n_{\mathrm{H}}+[(b+\epsilon) / h] \\
n_{\mathrm{H}, \mathrm{O}}(t)=[(b+\epsilon) / 2 f]\left(e^{2 f t}-1\right)=n_{\mathrm{H}}
\end{gathered}
$$

It is evident from these equations that for the time periods of interest $\left(t^{\prime} \gg 1\right)$ the $\mathrm{H}_{2} \mathrm{O}$ concentration will be identical with that of $\mathrm{H}$, the $\mathrm{O}$ concentration will be a very small constant fraction of the $\mathrm{H}$ concentration, and the $\mathrm{OH}$ concentration will be approximately the same as that of $O$. The form of these equations is, of course, directly attributable to the action of the chain, reactions VI, VII, and VIII. The equality of $n_{\mathrm{H}}$ and $n_{\mathrm{H}_{2} \mathrm{O}}$ is a consequence of the fastest reaction (VIII), which produces $\mathrm{H}_{2} \mathrm{O}$ at the same rate as $\mathrm{H}$.

Equations (11-18) are plotted in Fig. 1 for a temperature of $1500^{\circ} \mathrm{K}$ and for a stoichiometric mixture of hydrogenoxygen wherein the values for the kinetic constants were taken from Duff. The validity of these values will be discussed later in connection with ignition time delay. It is evident from the merging of the two forms of each radical equation that the action of the chain becomes a dominating factor before $t^{\prime}=10$. Thus the characteristic time has the physical significance of being a transition time between the important initiation reactions and the action of the chain.

\section{Ignition Time Delay}

It is now pertinent to define a time $\tau$, which is representative of the ignition delay. Once a sufficient number of hydrogen atoms have been produced (late in the induction period), the various reactions occur rapidly and exothermally to the degree that the temperature begins to increase. This increases the reaction rate exponentially so that the temperature soon increases exponentially. The "flame" is established

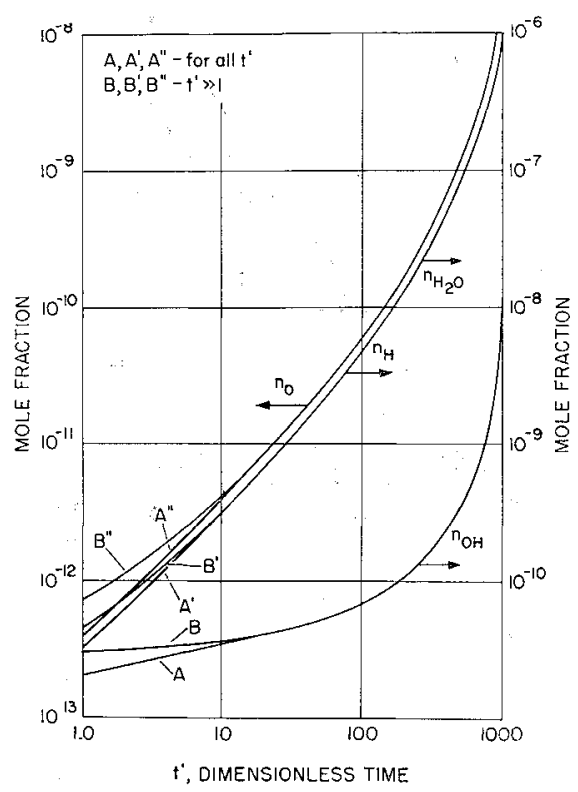

Fig. 1 Radical concentrations during the ignition delay period.

shortly thereafter. It would appear logical, then, to define $\tau$ as the time required for $n_{\mathrm{H}}$ to attain some suitably high value $\lambda$. The expression for ignition time delay can then be written directly from Eq. (15). That is,

$$
\tau=\frac{1}{2 f} \ln \left(\frac{2 f}{b+\epsilon} \lambda+1\right)
$$

Many equally valid criteria may be proposed for ascertaining $\lambda$, all of which lead to essentially the same value of $\tau$. However, it would appear consistent to arrive at a criterion based on the rate controlling reaction $\mathrm{H}+\mathrm{O}_{2} \stackrel{k_{6}}{\rightarrow} \mathrm{OH}+\mathrm{O}$, wherein account is taken of the decreasing oxygen concentration. Admittedly, the equations were derived under the assumption of constant $n_{\mathrm{O}_{2}}$, which is true to very good accuracy until the very last part of the delay zone. The results of the consequent linear theory will be used now to predict $n_{\mathrm{H}}(t)$ in the very late stages by including $n_{\mathrm{O}_{2}}(t)$. This correction now leads to an inflection point in $n_{\mathrm{H}}(t)$, which will be used as the criterion for predicting ignition time delay. Noting that $(b+\epsilon) 2 f$ is at most of the order of $10^{-6}$ in Eq. (15) and that $\lambda$ must be orders higher allows Eq. (15) to be written

$$
n_{\mathrm{H}}(t)=[(b+\epsilon) / 2 f] e^{2 f t}
$$

so that

$$
\dot{n}_{\mathrm{H}}=(b+\epsilon) e^{2 f t}=2 f n_{\mathrm{H}}=2 n_{\mathrm{O}_{\mathbf{2}}} n_{\mathrm{H}}[C] k_{6}
$$

In the very late stages of the delay zone, $n_{\mathrm{O}}$, decreases in accordance with Eq. (5) which, after dropping the small term, is

$$
\dot{n}_{\mathrm{O}_{2}}=-f n_{\mathrm{H}}=-\frac{1}{2} \dot{n}_{\mathrm{H}}
$$

This integrates to

$$
n_{\mathrm{O}_{2}}-\left(n_{\mathrm{O}_{2}}\right)_{0}=-\frac{1}{2} n_{\mathrm{H}}
$$

where now $n_{\mathrm{O}_{2}}$ is taken as time dependent and $\left(n_{\mathrm{O}_{2}}\right)_{0}$ is the initial value of $n_{\mathrm{O}_{\mathrm{y}}}$. Differentiating Eq. (20) and substituting Eq. (21) yields

$$
\ddot{n}_{\mathrm{H}}=2\left(n_{\mathrm{O}_{2}}\right)_{0} \dot{n}_{\mathrm{H}}[C] k_{6}-2 n_{\mathrm{E}} \dot{n}_{\mathrm{E}}[C] k_{6}
$$

It can be said arbitrarily that $\lambda$ will be that value of $n_{\mathrm{H}}$ corresponding to the inflection point in the $n_{\mathrm{H}}$ vs $t$ curve, that is, where $\dot{n}_{\mathrm{H}}$ is a maximum and $\ddot{n}_{\mathrm{E}}$ is 0 . Thus,

$$
\left(n_{\mathrm{H}}\right)_{\tau}=\lambda=\left(n_{\mathrm{O}_{2}}\right)_{0}
$$




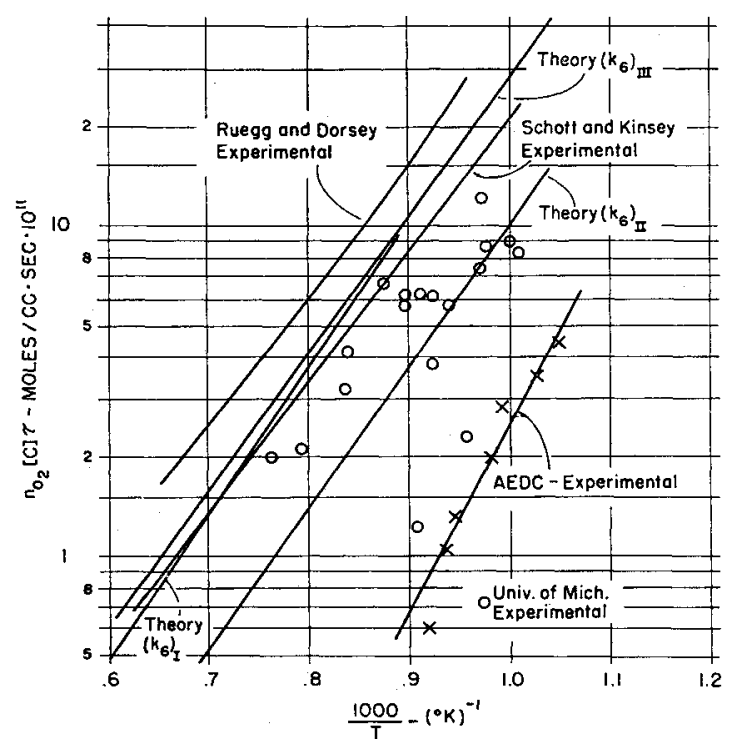

Fig. 2 Comparison of theoretical and experimental time delays for hydrogen-oxygen systems.

This $\lambda$ then defines a time representative of the ignition delay in a manner independent of the particular initial concentrations employed. As seen, the actual value of $\lambda$ is based on an initial concentration.

Substituting for $\lambda$ in Eq. (19) and noting that $(2 f / \epsilon) n_{\mathrm{O}_{2}} \gg$ 1 yields

$$
\tau=\frac{1}{2 f} \ln \frac{\left(2 f n_{\mathrm{O}_{2}}\right)}{b+\epsilon}=\frac{1}{2 n_{\mathrm{O}_{2}}[C] k_{6}} \ln \left[\frac{n_{\mathrm{O}_{2}}{ }^{2} k_{6}}{n_{\mathrm{B}_{2}} k_{2}+n_{\mathrm{H}_{2}} n_{\mathrm{O}_{2}} k_{5}}\right]
$$

where the subscript on $n_{\mathrm{O}_{2}}$ has been dropped, but it is to be understood that it represents the initial mole fraction. The logarithm term in Eq. (22) has slight but not negligible influence on $\tau$. Thus it is seen that the ignition time delay is dependent almost solely on the action of the chain. That is, the time required for the initiation processes to start the chain is negligible.

Fq. (22) can be rewritten, noting that $[C]=P / R T$ as

$$
\tau=\frac{R T}{2 n_{\mathrm{O}_{2}} P A_{6}} e^{E_{6} / R T} \ln \left[\frac{n_{\mathrm{O}_{2}}{ }^{2} k_{6}}{n_{\mathrm{H}_{2}} k_{2}+n_{\mathrm{B}_{2}} n_{\mathrm{O}_{2}} k_{5}}\right]
$$

The following conclusions can be drawn:

1) $\ln \tau$ is only approximately proportional to reciprocal temperature, wherein the slope is indicative of the activation energy of the controlling reaction.

2) $\tau$ is inversely proportional to pressure.

3) $\tau$ is approximately inversely proportional to the initial mole fraction of oxygen.

4) $\tau$ is weakly sensitive to $n_{\mathrm{H}_{2}}$.

5) $\tau$ is independent of the particular inert gas, being dependent only on the mole fraction of inert present.

The assumption was made throughout most of the analysis that $n_{\mathrm{O}_{2}}$ could be taken as a constant. The validity of this assumption insofar as it affects the prediction of ignition time delay can be justified through use of Eqs. (15) and (21) and the ignition delay criteria. A similar statement can be made and proven for the constancy of $n_{\mathrm{H}_{2}}$.

Patch $^{9}$ has considered the kinetics of the hydrogen-oxygendiluent system with special application to the prediction of composition limits. The lower temperatures encountered necessitated the inclusion of some reactions that were not important in the temperature range of interest in this study $\left(1100-2000^{\circ} \mathrm{K}\right)$. Conversely, he did not include many of the reactions considered in this paper. If one neglects the dissociation of hydrogen and the reactions involving $\mathrm{HO}_{2}$, Patch's prediction of the rate of growth of $\mathrm{H}$ is identical to that found in this paper for times much greater than the characteristic time.

\section{Comparison between Theoretical and Experimental Ignition Delays}

In Ref. 12, Nicholls compared the theoretical predictions of ignition time delay according to Eq. (22) with some experimentally determined values. This figure is repeated here as Fig. 2, in order to show the influence of the latest suggested values for the rate controlling reaction rate constant $k_{6}$. Included on the figure are the standing detonation wave results of Nicholls and Dabora, ${ }^{2}$ the shock tube results of Schott and Kinsey, ${ }^{6}$ the supersonic wind tunnel results of Rhodes and Chriss, ${ }^{13}$ and the ballistic range measurements of Ruegg and Dorsey.14 A plot of Eq. (22) is superimposed for three different values of $k_{6}$. The value of $\left(k_{6}\right)_{1}$, given by Duff, ${ }^{5}$ is

$$
\left(k_{6}\right)_{\mathrm{I}}=4 \times 10^{14} \exp (-18 / R T)\left(\operatorname{moles} / \mathrm{cm}^{3}\right)^{-1} \mathrm{sec}^{-1}
$$

Very recently Kaufman and Del Greco ${ }^{15}$ suggested

$\left(k_{6}\right)_{\mathrm{II}}=1.7 \times 10^{16} T^{-0.37} \exp (-18.1 / R T)\left(\text { moles } / \mathrm{cm}^{3}\right)^{-1} \times$

for the temperature range $300-2500^{\circ} \mathrm{K}$. Baldwin, ${ }^{16}$ in discussing Kaufman and Del Greco's paper, suggests

$$
\left(k_{6}\right)_{\mathrm{III}}=10^{14.33 \pm 0.3} \exp \left(-\frac{16.6 \pm 0.8}{R T}\right)\left(\frac{\text { moles }}{\mathrm{cm}^{3}}\right)^{-1} \mathrm{sec}^{-1}
$$

over the temperature range $293-1500^{\circ} \mathrm{K}$. It can be noted that the value of $\left(k_{6}\right)_{\mathrm{I}}$ and the mean value of $\left(k_{6}\right)_{\mathrm{III}}$ are in good agreement, whereas $\left(k_{6}\right)_{\text {II }}$ predicts substantially lower values of time delay (which, to good approximation, is proportional to the inverse of $k_{6}$ ). The results of Schott and Kinsey are in good agreement with $\left(k_{6}\right)_{1}$ and $\left(k_{6}\right)_{11 I}$, whereas the University of Michigan results span the region of uncertainty in $k_{6}$. Relative to $\left(k_{6}\right)_{\mathrm{m}}$ Baldwin states, "the estimates of error are generous and allow a $50 \%$ error in both high and low temperature values."

When one considers that the experimental data are subject to appreciable scatter, that no allowance is made for vibrational relaxation effects, and that ignition time delay is not a uniquely defined quantity, one can hardly draw a conclusion, based on ignition time delay, as to the relative merits of these different values of $k_{6}$.

Finally it is of interest to compare the theoretical predictions of ignition time delay according to Eq. (22) with the theoretical results of Momtchiloff, Taback, and Buswell, ${ }^{17}$ who numerically integrated the equations of motion and the kinetic equations for the flow of hydrogen-air in a constant area channel. Using their value for $k_{6}\left[5.64 \times 10^{13}\right.$ $\exp (-15.1 / R T)]$, Eq. (22) predicts times about 1.5 to 2 times larger. The agreement is considered good in that part of this difference is attributable to different definitions of ignition time delay, and, further, the difference is much less than the present uncertainty in $k_{6}$.

\section{References}

I Nicholls, J. A., "Stabilization of gaseous detonation waves with emphasis on the ignition time delay zone," $\mathrm{Ph} . \mathrm{D}$. Thesis, Univ. Mich. (February 1960); also Air Force Office Sci. Res. TN 60-442 (1960)

2 Nicholls, J. A. and Dabora, E. K., "Recent results on standing detonation waves," 8th (International) Symposium on Combustion (The Williams and Wilkins Company, Baltimore, Md., 1962), pp. 644-655; also Air Force Office Sci. Res. TN 60-441 (1960).

${ }^{3}$ Semenov, N. N., Some Problems in Chemical Kinetics and Reactivity (Princeton University Press, Princeton, N. J., 1958), Vol. II.

${ }^{4}$ Lewis, B. and von Elbe, G., Combustion, Flames and Explosions of Gases (Academic Press, New York, 1951), pp. 579-627.

5 Duff, R. E., "Calculation of reaction profiles behind steadystate shock waves. I. Application to detonation waves," J. Chem. Phys. 28, 1193-1196 (1958).

${ }^{6}$ Schott, G. L. and Kinsey, J L., "Kinetic studies of hydroxyl 
radicals in shock waves. II. Induction times in the hydrogenoxygen reaction," J. Chem. Phys. 29, 1177-1182 (1958).

7 Bethe, H. A. and Teller, E., "Deviations from thermal equilibrium in shock waves," Rept. X-117, Ballistic Res. Lab., Aberdeen Proving Ground (1945)

${ }^{8}$ Wood, G. P., "Calculations of the rate of thermal dissociation of air behind normal shock waves at Mach numbers of 10, 12, and 14,"NACA TN 3634 (April 1956).

'Patch, R W., "Prediction of composition limits for detonation and hydrogen-oxygen-diluent mixtures," ARS J. 31, 46-51 (1961)

${ }^{10}$ Nicholls, J. A., Dabora, E. K., and Gealer, R. L, "Studies in connection with stabilized detonation waves," Seventh Symposium on Combustion (Butterworths Scientific Publications, London, 1959), p. 766.

11 Nicholls, J. A., Dabora, E. K., Morrison, R. B., Adamson, T. C., Jr., Phillips, R. L., Ranger, A. A., Lelli, G. J., and Cosens, G. L., "An experimental and theoretical study of stationary gaseous detonation waves," Air Force Office Sci. Res. TN
1764 (October 1961).

12 Nicholls, J. A., "Standing detonation waves," Ninth (International) Symposium on Combustion (Academic Press, New York, 1963), pp. 488-496.

${ }^{13}$ Rhodes, R. P. and Chriss, D. E., "A preliminary study of stationary shock induced combustion with hydrogen-air mixtures," Arnold Eng. Dev. Center TN-61-36 (July 1961).

${ }^{14}$ Ruegg, F. W. and Dorsey, W. W., "A missile technique for the study of detonation waves," J. Res., Natl. Bur. Std. 66C, 51-58 (1962)

${ }^{15}$ Kaufman, F. and Del Greco, F. P., "Fast reactions of $\mathrm{OH}$ radicals," Ninth Symposium (International) on Combustion (Academic Press, New York, 1963), pp. 659-666.

${ }_{16}$ Baldwin, R. R. discussion of Ref. 15, p. 667 .

${ }_{17}$ Momtchiloff, I. N., Taback, E. D., and Buswell, R. F., "Kinetics in hydrogen-air flow systems. I. Calculation of ignition delays for hypersonic ramjets," Ninth Symposium (International) on Combustion (Academic Press, New York, 1963), pp. 220-230.

\title{
Geometrical Construction of Two-Dimensional and Axisymmetrical Flow Fields
}

\author{
Friedrich O. Ringleb* \\ Naval Air Engineering Center, Philadelphia, $P a$.
}

\begin{abstract}
It is assumed that a potential flow field, consisting of lines of constant velocity potential and of streamlines, is approximated by a net of circular ares that intersect at right angles. In general, this is possible with considerably greater accuracy than by using a net of straight elements with the same number of meshes. The method of constructing such a field, as derived in the following, is based on a theorem of elementary geometry which seems to have been unknown or at least has not been used in the field of fluid mechanies. It states that the corners of a rectangle formed by circular ares are situated on a circle. A proof is given in this paper by conformal mapping. This method can be applied not only to two-dimensional but also to axisymmetrical flow of incompressible or compressible media. Its simplicity and the small overall number of steps generally required permit the solution of problems with boundary conditions where iteration is necessary. The paper discusses various examples and demonstrates the accuracy of the method in cases where an exact solution is known.
\end{abstract}

\section{Nomenclature}

\author{
$=$ velocity potential \\ $=$ stream function \\ $=$ arc element of a streamline \\ $=$ arc element of an equal potential line \\ $=$ velocity of flow \\ $=$ angle of flow direction with a fixed direction \\ $=$ distance from axis of symmetry \\ $=$ mass density \\ $=$ pressure \\ $=$ absolute temperature \\ $=$ velocity of sound \\ $=$ Mach number \\ $c_{p} / c_{v}=$ ratio of specific heats
}

$c, \rho, p$ for the gas at rest $(w=0)$.

Presented at the ARS 17 th Annual Meeting and Space Flight Exposition, Los Angeles, Calif., November 13-18, 1962; revision received June 21, 1963.

* Staff Physicist, Naval Air Engineering Laboratory; also Professor of Mechanical Engneering, University of Delaware, Newark, Del. Member AIAA.

\section{Introduction}

CEOMETRICAL methods of a stepwise character have not been applied very frequently in fluid mechanics and usually have been considered as laborious and rather inaccurate (see Ref. 1, p. 133), except perhaps the method of characteristics as used in gasdynamics for the solution of supersonic problems (refer to the list of textbooks on gasdynamics given in Ref. 2). On the other hand, the analytical methods also are usually laborious and, up to the present time, have not provided the solutions to a number of very interesting and important problems.

The lack of accuracy of stepwise geometrical flow construction methods usually stems from the approximation of equipotential lines and streamlines by piecewise straight elements. In order to increase the accuracy, it is necessary to increase the number of steps, in which case both the number of errors and the laboriousness of the procedure are increased. Therefore, it seemed to be appropriate to develop a method where the equipotential lines and the streamlines are approximated piecewise by circular arcs in such a way that the meshes of the net of these lines are replaced by rectangles 